


\begin{tabular}{llll}
\multirow{3}{*}{ Johan Verbraecken } & $\square$ & Dept of Pulmonary \\
& Dept of Pulmonary & Medicine and \\
& Multidisciplinary Sleep & Multidisciplinary Sleep \\
& Disorders Centre, Antwerp & Disorders Centre, Antwerp \\
& University Hospital and & University Hospital and \\
University of Antwerp, & University of Antwerp, \\
& Antwerp, Belgium & Wilrijkstraat 10, B-2650 \\
& Edegem, Antwerp, Belgium
\end{tabular}

\section{Complex sleep apnoea syndrome}

\section{Summary}

Complex sleep apnoea syndrome (CompSAS) is a type of central apnoea characterised by the development or persistence of central apnoeas or hypopnoeas during application of continuous positive airway pressure (CPAP; central apnoea index $\geqslant 5$ events $\left.\cdot h^{-1}\right)$. These patients present with predominantly obstructive apnoeas during diagnostic sleep studies. No clinical characteristics have been identified which can distinguish them from patients with a normal response during CPAP titration. Probably, this pattern is provoked by the application of high CPAP pressures in obstructive sleep apnoea syndrome patients with enhanced chemoreceptor sensitivity, or in patients with pronounced sleep fragmentation. Negative feedback is sent to the respiratory centre, via the Hering-Breuer reflex, which can lead to central apnoea. However, these explanations remain hypothetical at present. In a broader perspective, any occurrence of significant central apnoea activity during attempts to restore airway patency could be classified as CompSAS. Treatment modalities consist of prevention on the one hand (avoidance of high pressures and permissive flow limitation), and advanced ventilation techniques (adaptive servo ventilation) on the other hand.

\section{Introduction}

Snoring and obstructive sleep apnoea syndrome (OSAS) are the most common manifestations within a group of disorders described as "sleep disordered breathing"
$[1,2]$. To a lesser extent, central sleep apnoea syndrome (CSAS) can also be present, characterised by a decreased central respiratory drive during sleep, resulting in decreased or absent ventilation and disturbed gas exchange [3]. In OSAS, repetitive collapse of the upper airway takes place, which will finally
Statement of Interest None declared.
(C)

HERMES syllabus link module: B.19 
lead to $\mathrm{O}_{2}$ desaturation and arousal. Continuous positive pressure ventilation (CPAP) is the standard therapy to keep the airway open. The optimal pressure is determined during a supervised CPAP pressure titration night with polysomnographic monitoring in a sleep centre [4]. In most OSAS patients, CPAP will improve nocturnal breathing pattern. However, a small group of OSAS patients develop central apnoeas during CPAP titration. This category of sleep disordered breathing is currently defined as complex sleep apnoea syndrome (CompSAS) [5].

\section{Definition}

CompSAS can be defined as a sleep apnoea syndrome that initially is diagnosed as OSAS, but is characterised by the frequent occurrence of central apnoeas during CPAP therapy, after elimination of obstructive events (fig. 1) $[5,6]$. In the literature, CompSAS has been described as CPAP-related periodic breathing [7], complex disturbed breathing during sleep [8], CPAP-related CSA [9], CSA during CPAP: "CPAP-emergent CSA", i.e. was not present on diagnostic polysomnography (PSG); CPAPpersistent CSA (i.e. was also present on diagnostic PSG); "CPAP-induced CSA" [6]; refractory sleep apnoea [10]; and residual sleep apnoea [11]. However, the term "CPAP-resistant CSA" is used in those with pre-existent CSA [12].

\section{Pathogenesis}

The pathogenesis of CompSAS is not known and rarely studied, but in general, it is assumed that the presence of upper airway collapse, combined with a heavily disturbed ventilatory control is causative $[13,14]$.

The first hypothesis claims that an altered $\mathrm{CO}_{2}$ excretion takes place when upper airway obstruction is released by CPAP. The permanent open upper airway results in an improved gas exchange, which will lead to a decrease in arterial carbon dioxide partial pressure $\left(\mathrm{PaCO}_{2}\right)$. When this value drops below the apnoeic threshold, a central apnoea will develop $[15,16]$.

A second hypothesis is that over-titration with CPAP will lead to the development of central apnoeas [9, 17]. How this process exactly takes place is not well understood, but a potentially important factor could be the activation of stretch receptors in the lungs. When these receptors are activated due to an excessive expansion of the lungs, they send signals to the breathing centre along the vagal nerve, which inhibits the central motor output. Hence, this will result in an interruption of
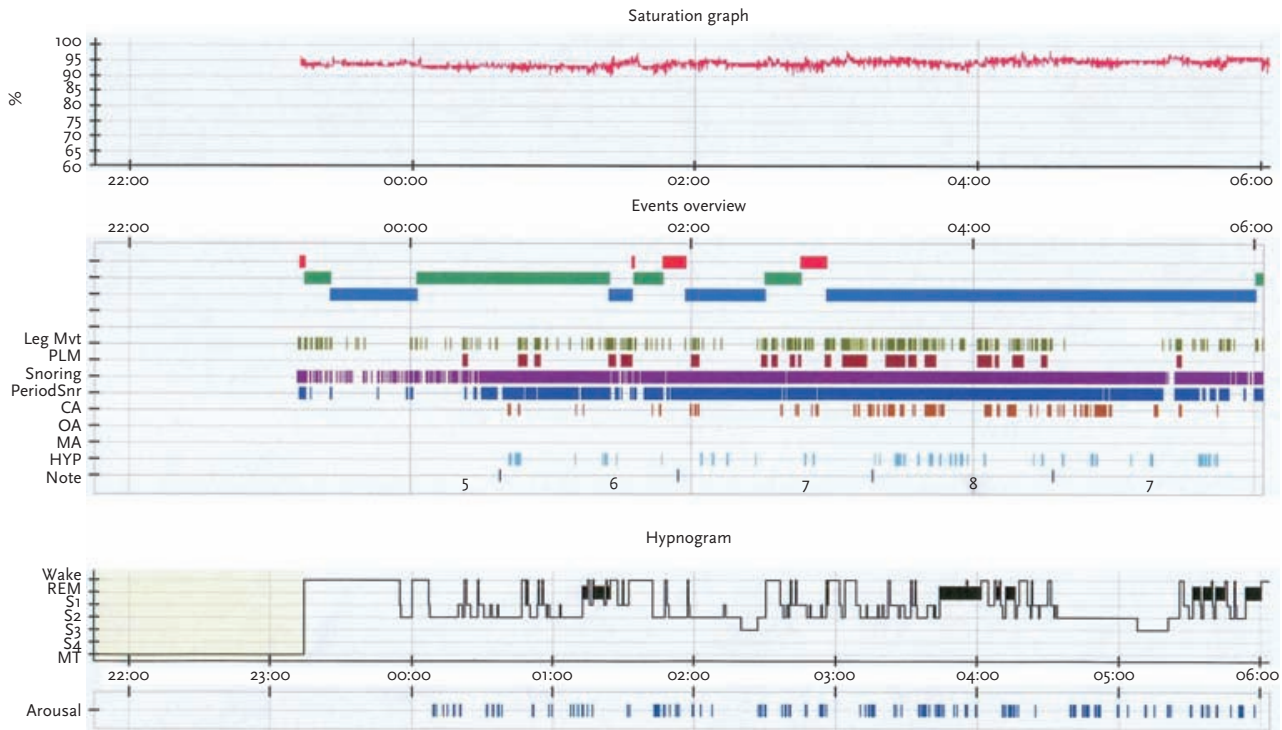

Figure 1

Overview of a CPAP titration night in an OSAS patient that develops central apnoeas. From top to bottom: oxygen saturation, sleep position, heart frequency, leg movement (Leg Mvt; PLM: periodic leg movement), snoring intensity, apnoeas (CA: central apnoea; OA: obstructive apnoea; MA: mixed apnoea), hypopnoeas (HYP), hypnogram. 
inspiration. This mechanism protects the lungs against overexpansion, and is called the Hering-Breuer reflex.

A final hypothesis is based on the fact that CPAP will worsen sleep quality in some patients $[16,18,19]$. The frequent changes from sleep to wake and from wake to sleep lead to instability in the ventilatory control system. This leads to oscillations in the $\mathrm{PaCO}_{2}$ values that can lead to a decrease in $\mathrm{PaCO}_{2}$ below the apnoeic threshold, with inherent central apnoeas as a consequence. It remains unclear to what extent sleep impairment is the cause or the effect of CompSAS [20]. It was suggested that an elevated nasal resistance might relate to frequent arousals, which could presumably contribute to an increase in central apnoeas [21]. This argues for a causal relationship.

A counter-argument could be that sleep in OSAS is already fragmented, even before CPAP is initiated, but that CPAP probably leads to an increase in the hypoxia-induced hyperventilation, through a decrease in pharyngeal resistance and an increase in ventilatory response on arousals. However, there are indications that sleep fragmentation in untreated CompSAS is more pronounced than in conventional OSAS [6]. This finding favours a predetermined as opposed to an iatrogenic cause for the development of CompSAS. Although the exact mechanism of CompSAS is unknown, probably several mechanisms operate together to produce the CompSAS phenotype. This multitude of factors contributing to CompSAS is the probable reason for variable behaviour of CompSAS patients during acute testing and longitudinal therapy with CPAP in different patient populations [22]. The use of bilevel continuous airway pressure (BiPAP) can also lead to CompSAS [23]. BiPAP can be used as an alternative for CPAP, in case of CPAP intolerance, or when CompSAS is observed during a CPAP titration night. The inspiratory positive pressure can lead to increased tidal volumes, which leads to a decrease in $\mathrm{PaCO}_{2}$. As mentioned earlier, central apnoeas will develop when $\mathrm{PaCO}_{2}$ is rendered below the apnoeic threshold.

\section{Prevalence}

In recent years, different studies have reported the prevalence of CompSAS to be between 0.56 and $18 \%$. High prevalences were reported by an Italian [7] and two US studies [5, 24] in which $15 \%$ and $18 \%$ of the patients developed central apnoeas. The highest prevalence (18\%) was reported in series of patients with chronic heart failure and OSAS [24]. The lowest prevalence $(0.56 \%)$ was reported in a German study measuring brain natriuretic peptide (BNP) as an additional marker for the exclusion of possible confounding Cheyne-Stokes respiration cases [25].

An Australasian study [6] reported $13.1 \%$, while two Japanese studies reported a prevalence of $5 \%[18,19]$. In a Belgian study, CompSAS was found in only nine $(2.9 \%)$ out of 349 CPAP titration nights for OSAS [26]. A US study reported CompSAS in $6.5 \%$ of their patients in an extensive series of 1286 CPAP titrations [12], while a German study reported a prevalence of $12.4 \%$ during titration, and $6.9 \%$ during follow-up $3.2 \%$ in those with initial CompSAS and $3.7 \%$ de novo CompSAS) [20]. Differences in prevalence data are attributed to fast CPAP titration (that may lead to higher pressures and induce central apnoeas), supine position [27], sleep duration, use of split-night designs [5], intake of opioid medication [5], ethnic differences [28] and presence of predisposing factors for chronic heart failure [5]. The exclusion of these factors is especially important for patients with a history of coronary heart disease, hypertension, and/or atrial fibrillation [29]. It must also be emphasised that airway obstruction, especially in the supine position, can mimic central apnoea [30], while the high prevalence of supine dependency of CompSAS by THOMAS et al. [27] was intended, as patients were examined predominantly in the supine position [27]. Overall, the prevalence of CompSAS in clinical settings remains somewhat hazy, while the multitude of pathways leading to CompSAS results in a dynamic phenotype, with the improvement or disappearance of CompSAS activity in some patients, and de novo appearance in others.

\section{Diagnostics}

CompSAS is diagnosed based on PSG. The first condition is the presence of OSAS, based on a minimal apnoea-hypopnoea index (AHI) of 5 events per hour of sleep with a majority of obstructive events. If during CPAP titration, a reduction is obtained of the number of obstructive events to $<_{5}$ events per hour of 
sleep, while the central apnoea index (CAI) is $\geqslant 5$ events per hour sleep, the diagnosis of CompSAS can be established [6, 31]. Research has indicated that CompSAS can be distinguished from OSAS by means of an automated electrocardiography-based method during a diagnostic sleep study. This application is however not yet available in the routine setting [32].

\section{Clinical presentation}

Most studies show that more than $80 \%$ of CompSAS patients are male. An explanation could be that males have a higher hypercapnic ventilatory response, and are therefore more predestinated to develop CompSAS. Not all studies came to the same conclusion [19]. In general, patients with OSAS or CompSAS do not show statistically significant differences in age, level of sleepiness (based on Epworth scale) $[5,9,11,31]$, lung function parameters (i.e. forced expiratory volume in 1 s) [9] or arterial blood gases. One of the characteristics of OSAS patients is an elevated body mass index (BMI), which is also observed in CompSAS [5, 6, 9, 18], although some authors have found a somewhat lower BMI [31]. One study reported a BMI higher than in conventional OSAS patients [19]. Different studies also reported the severity of OSA as one of the risk factors for long-term CPAP-persistent central apnoeas [12, 33].

Given the frequent association of central apnoea and chronic heart failure (CHF) [3], the prevalence of $\mathrm{CHF}$ and ischaemic heart disease (IHD) has also been studied in CompSAS. However, no consistent findings were reported $[6,9,19,31]$. Recently, it was reported that only one-third of patients with CompSAS had any risk factor of CompSAS (arterial hypertension, coronary artery disease, CHF) prior to sleep testing [28]. Last but not least, elevated nasal resistance was also associated with CompSAS [21].

\section{Polysomnographic characteristics a priori}

Based on the diagnostic PSG to establish OSAS, it is not possible to predict whether someone will present with CompSAS. Some evidence has been reported that CompSAS patients might have a higher CAl at diagnosis than OSAS patients (CAI $>5$ events per hour in $46 \%$ of CompSAS compared with $8 \%$ of OSAS), but this could not be confirmed by others [5, 6, 31]. One study reported a significantly higher CAI in non-rapid eye movement (NREM) sleep and in supine position, but the differences were small and not clinically relevant [19]. During CPAP titration, CompSAS patients present central apnoeas most often during NREM sleep, and certainly much fewer or no apnoeas during REM sleep [9, 34]. A higher arousal index was reported in CompSAS in one study [6], but was not confirmed in other studies $[12,19]$.

\section{Use of BNP}

There are arguments for choosing BNP levels as a criterion for detection or exclusion of heart failure in patients with CompSAS [25]. Concomitant heart failure may trigger susceptibility to central apnoeas (so-called CheyneStokes respiration), and may influence prevalence data. Moreover, the occurrence of Cheyne-Stokes respiration is not dependent on reduced systolic left ventricular function. Cheyne-Stokes respiration can also be seen in patients with diastolic heart failure or only mildly reduced systolic left ventricular function. Therefore, an ejection fraction cut-off of $40 \%$ is not sensitive enough to select patients with chronic heart failure and Cheyne-Stokes respiration $[29,35]$. Finally, identification of $\mathrm{CHF}$ can allow for better tailoring of therapy to pathophysiology involved in an individual patient. Patients with elevated BNP $\left(>100 \mathrm{pg} \cdot \mathrm{mL}^{-1}\right)$ can then be excluded. However, it has to be borne in mind that BNP can also be influenced by other diseases (renal failure, liver cirrhosis), medication or obesity.

\section{Treatment of CompSAS}

\section{Prevention}

Rather than starting specific treatments, it is useful to consider whether high CPAP pressures are applied (pressure toxicity), provoking CompSAS.

Patients with CompSAS most often respond well to positive airway pressure, but the obstruction cannot always be eliminated without aggravating periodic breathing or provoking 
central apnoeas. A possible preventive measure is the so-called permissive flow limitation [8]. The pressure is set on a level that keeps a mild degree of airway obstruction, without disturbing ventilatory control mechanisms. When $\mathrm{O}_{2}$ desaturations occur with central apnoeas during CPAP application, combined oxygen administration can be considered. Patients report that this helps to improve sleep quality, although this is probably a placebo effect [8]. However, $\mathrm{O}_{2}$ administration will lead to a decrease of the hypoxic ventilatory response, which stabilises breathing, and CompSAS will disappear [36]. Finally, drugs that induce breathing instability, like methadone and baclofen, have to be avoided [8].

\section{Pharmacotherapy}

When prevention is not sufficient, pharmacotherapy can be considered. Theophylline and acetazolamide can contribute to an improvement in periodic breathing. However, these drugs are not used as a single therapy, due to their limited effect and eventual side-effects [37]. Stabilisation of NREM sleep can also be useful by means of adequate sleep hygiene, regular sleep-wake times, and by use of traditional sleeping pills (benzodiazepines) and sleep stabilising antidepressants (trazolan, mianserin) [8].

\section{Expected evolution when CPAP therapy is maintained}

A few studies have shown that in the majority of patients with CompSAS, central apnoeas disappear (almost) completely during followup PSG [9], with improvement in sleep efficiency, a decrease in wake after sleep onset, and a lower number of arousals in comparison with the baseline diagnostic night. However, a number of studies reported a minor effect of CPAP on sleep and breathing variables [38]. Therefore, CPAP seems effective in some patients, while ineffective in others. CompSAS also seems to develop de novo in $4 \%$ of OSAS patients during follow up for CPAP therapy [20]. Very few data are available regarding CPAP compliance and clinical improvement. In one study, CompSAS patients demonstrated more dyspnoea, poor CPAP adherence and more often spontaneous removal of the mask [31]. Another study reported an inferior effect of CPAP on sleep as well as breathing parameters [39].

\section{BiPAP in the spontaneous-timed mode and adaptive servoventilation}

An effective treatment of CompSAS is possible with supporting ventilation during central apnoeas and avoidance of $\mathrm{PaCO}_{2}$ oscillations. This can be realised with the help of BiPAP in the spontaneous-timed (ST) mode or by means of adaptive servoventilation (ASV) $[8,39,40]$. BPAP-ST is a type of noninvasive ventilation that offers a pneumatic splint effect in the airways, supporting ventilation and initiating breathing during episodes of apnoeas. The expiratory positive airway pressure (EPAP) is set to eliminate apnoeas, while the inspiratory positive airway pressure (IPAP) and back-up ventilation frequency (ST mode) are set in order to decrease hypoventilation and prevent overcompensation that leads to hypocapnia and hyperventilation [36].

With ASV, the end expiratory pressure is determined during PSG by evaluating the pressure needed to eliminate obstructive apnoeas. The ASV device automatically determines the extent of the ventilatory support during inspiration (within a set pressure range) based on continuous analysis of the breathing pattern during inspiration $[35,41]$. Recently, advanced ASV machines have been developed that include an automatic expiratory pressure adjustment, an advanced algorithm for distinguishing open versus obstructed airway apnoea, a modified auto backup rate which is proportional to subject's baseline breathing rate, and a variable inspiratory support [42].

Some trials have evaluated the efficiency of ASV versus other treatments in CompSAS. ASV gave a superior decrease of $\mathrm{AHI}$ and $\mathrm{CAI}$ versus CPAP or BiPAP-ST $[43,44]$. Moreover, a better sleep quality and symptomatic improvement was reported by $72 \%$ during follow-up, as well as a global excellent tolerance for ASV. In a study comparing ASV with BiPAP-ST, both ventilatory modalities resulted in a decrease of the $\mathrm{AHI}$ and respiratory arousal index, but only ASV was able to reduce these parameters to almost zero [39]. AHI could be normalised with ASV $\left(<5\right.$ events $\left.\cdot h^{-1}\right)$ in the majority of CompSAS patients. RANDERATH et al. [45] evaluated the effect of ASV in 
patients with obstructive as well as central apnoeas. The total $\mathrm{AHI}$ decreased from 46.8 to 7.8 , the central $\mathrm{AHI}$ from 25.7 to 7.1 and the obstructive $\mathrm{AHI}$ from 14.2 to 0.7 . Also sleep quality was significantly better after treatment with ASV. Based on these results, it can be concluded that positive airway pressure, and predominantly ASV, is very effective in CompSAS.

\section{$\mathrm{CO}_{2}$ administration}

A last treatment modality for CompSAS is the combined approach of CPAP or BiPAP with $0.5-1 \% \mathrm{CO}_{2}$, resulting in an immediate $(<1 \mathrm{~min})$ decrease of $\mathrm{AHI}$ to $<5$ events $\cdot \mathrm{h}^{-1}$, without complaints of dypnoea, palpitations or headache $[35,46]$. Administration of low concentrations of $\mathrm{CO}_{2}$ during CPAP or BiPAP therapy therefore seems extremely effective in CompSAS, since administration of $\mathrm{CO}_{2}$ keeps the prevailing $\mathrm{PaCO}_{2}$ above the apnoeic threshold. Moreover, increased fractional concentration of inspired $\mathrm{CO}_{2}$ has an airway resistance-lowering effect in sleeping subjects with high airway resistance, which may minimise the level of CPAP pressure required to ensure airway patency in OSA patients, presumably because of chemoreceptor activation of motor neurons serving both upper airway and pump muscles [47, 48].

Safety concerns of gas application have to be addressed before the application of $\mathrm{CO}_{2}$ can be considered for clinical use, while increase in sympathetic activity has been observed. Enlargement of dead space or enhanced expiratory rebreathing space in positive airway pressure therapy has been shown to be effective as well in the treatment of CompSAS [49, 50,51]. A small volume of $\mathrm{CO}_{2}$ is trapped and inhaled, resulting in a transient increase in inspired $\mathrm{CO}_{2}$ that ultimately reaches the peripheral and central chemoreceptors.

\section{CPAP-independent CompSAS}

Recently, there have been arguments to label this form of sleep disordered breathing as CompSAS in the absence of a CPAP trial, since emerging central apnoeas were also reported after initiation of oral appliance [52], tracheostomy [53], maxillofacial surgery [54] and after surgical relief of nasal obstruction [55].

CPAP-independent factors, such as sleep fragmentation induced by (any) therapy, reduction of $\mathrm{PaCO}_{2}$ and CPAP-independent tendency towards periodic breathing could also be involved in producing the CompSAS phenotype. Consistent with the evolution reported during CPAP therapy in two tracheotomy studies in which OSA patients underwent serial PSGs, it was shown that OSA patients who underwent therapeutic tracheotomy initially had central apnoeas, which decreased later on a repeat PSG $[56,57]$. Anyway, obstructive cycles are replaced by central ones with similarly disrupted sleep and poor therapy compliance and clinical outcomes are not surprising.

\section{Conclusion}

CompSAS is a disorder in patients with OSAS, who develop central apnoeas during CPAP titration. The pathogenesis is probably based on disturbed ventilatory control. The prevalence of CompSAS varies between $2.6 \%$ and $15 \%$ and the patients are most often male. There are indications that CompSAS patients have more sleep fragmentation during the diagnostic sleep study, have a higher prevalence of congestive heart failure and/or ischaemic heart disease, but the literature is not conclusive. CompSAS is probably a transient problem, which disappears spontaneously during chronic CPAP therapy. Best treatment results are obtained with ASV. an American Academy of Sleep Medicine Task Force. Sleep 1999; 22: 667-689.

3. Verbraecken J, De Backer W. Upper airway mechanics. Respiration 2009; 78: 121-133.

4. Kushida CA, Littner MR, Hirshkowitz M, et al. Practice parameters for the use of continuous and mendations for syndrome definition and measurement techniques in clinical research. The Report of
. Young T, Palta M, Dempsey J, et al. The occurrence of sleep-disordered breathing among middle-aged adults. N Engl J Med 1993; 328: 1230-1235.

2. Sleep-related breathing disorders in adults: recom 
bilevel positive airway pressure devices to treat adult patients with sleep-related breathing disorders. Sleep 2006; 29: 375-380.

5. Morgenthaler TI, Kagramanov V, Hanak V, et al. Complex sleep apnea syndrome: is it a unique clinical syndrome? Sleep 2006; 29: 1203-1209.

6. Lehman S, Antic N, Thompson C, et al. Central sleep apnea on commencement of continuous positive airway pressure in patients with a primary diagnosis of obstructive sleep apnea-hypopnea. J Clin Sleep Med 2007; 3: 462-466.

7. Marrone O, Stallone A, Salvaggio A, et al. Occurrence of breathing disorders during CPAP administration in obstructive sleep apnea syndrome. Eur Respir J 1991; 4: 66o-666.

8. Gilmartin GS, Daly RW, Thomas RJ. Recognition and management of complex sleep-disordered breathing. Curr Opin Pulm Med 2005; 11: 485-493.

9. Dernaika T, Tawk M, Nazir S, et al. The significance and outcome of continuous positive airway pressurerelated central sleep apnea during split-night sleep studies. Chest 2007; 132: 81-87.

10. Badr MS, Grossman JE, Weber SA. Treatment of refractory sleep apnea with supplemental carbon dioxide. Am J Respir Crit Care Med 1994; 150: 561-564.

11. Mulgrew AT, Lawati NA, Ayas NT, et al. Residual sleep apnea on polysomnography after 3 months of CPAP therapy: clinical implications, predictors and patterns. Sleep Med 2010; 11: 119-125.

12. Javaheri S, Smith S, Chung E. The prevalence and natural history of complex sleep apnea. J Clin Sleep Med 2009; 5: 205-211.

13. Marrone O. Complex sleep apnea and obesity hypoventilation syndrome. Opposite ends of the spectrum of obstructive sleep apnea? Med Hypotheses 2009; 73: 488-492.

14. Salloum A, Rowley JA, Mateika JH, et al. Increased propensity for central apnea in patients with obstructive sleep apnea. Effect of nasal continuous positive airway pressure. Am J Respir Crit Care Med 2010; 181: 189-193.

15. Gay P. Complex sleep apnea: it really is a disease. J Clin Sleep Med 2008; 4: 403-405.

16. Malhotra A, Bertisch S, Wellman A. Complex sleep apnea: it isn't really a disease. J Clin Sleep Med 2008; 4: 406-408.

17. Brown LK, Casey KR. Complex sleep apnea: the hedgehog and the fox. Curr Opin Pulm Med 2007; 13: 473-478.

18. Endo $Y$, Suzuki $M$, Inoue $Y$, et al. Prevalence of complex sleep apnea among Japanese patients with sleep apnea syndrome. Tohoku J Exp Med 2008; 215: 349-354.

19. Yaegashi H, Fujimoto K, Abe $\mathrm{H}$, et al. Characteristics of Japanese patients with complex sleep apnea syndrome: a retrospective comparison with obstructive sleep apnea syndrome. Intern Med 2009; 48: 427-432.

20. Cassel W, Canisius S, Becker HF, et al. A prospective polysomnographic study on the evolution of complex sleep apnoea. Eur Respir J 2011; 38: 329-337.

21. Nakazaki C, Noda A, Yasuda Y, et al. Continuous positive airway pressure intolerance associated with elevated nasal resistance is possible mechanism of complex sleep apnea syndrome. Sleep Breath 2012; 16: $747-752$.

22. Kuzniar TJ, Kasibowska-Kuzniar K, Freedom T. Trials of bilevel positive airway pressure-spontaneous in patients with complex sleep apnoea. Pneumol Allergol Pol 2012; 80: 214-219.

23. Johnson KG, Johnson DC. Bilevel positive airway pressure worsens central apneas during sleep. Chest 2005; 128: 2141-2150.
24. Bitter T, Westerheide N, Hossain MS, et al. Complex sleep apnoea in congestive heart failure. Thorax 2011; 66: 402-407.

25. Westhoff M, Arzt M, Lietterst P. Prevalence and treatment of central sleep apnoea emerging after initiation of continuous positive airway pressure in patients with obstructive sleep apnoea without evidence of heart failure. Sleep Breath 2012; 16: 71-78.

26. Verbraecken J, Schoonis L, Verplancke B, et al. Prevalence of complex sleep apnea (CompSAS) during manual CPAP titration for obstructive sleep apnea. Eur Respir J 2009; 34: Suppl. 53, 649s.

27. Thomas RJ, Terzano MG, Parrino L, Weiss JW. Obstructive sleep-disordered breathing with a dominant cyclic alternating pattern - a recognizable polysomnographic variant with practical clinical implications. Sleep 2004; 27: 229-234.

28. Kuzniar TJ, Kasibowska-Kuzniar K, Ray DW, et al. Clinical heterogeneity of patients with complex sleep apnea syndrome. Sleep Breath 2013 [In press; DOI: 10.1007/s11325-013-0825-4].

29. Westhoff M, Arzt M, Litterst P. Influence of adaptive servoventilation on B-type natriuretic petide in patients with Cheyne-Stokes respiration and mild to moderate systolic and diastolic heart failure. Pneumologie 2010; 64: 467-473.

30. Vanderveken OM, Oostveen E, Boudewyns AN, et al. Quantification of pharyngeal patency in patients with sleep-disordered breathing. J Otorhinolaryngol Relat Spec 2005; 67: 168-179.

31. Pusalavidyasagar SS, Olson EJ, Gay PC, et al. Treatment of complex sleep apnea syndrome: a retrospective comparative review. Sleep Med 2006; 7: 474-479.

32. Thomas RJ, Mietus JE, Peng CK, et al. Differentiating obstructive from central and complex sleep apnea using an automated electrocardiogram-based method. Sleep 2007; 30: 1756-1769.

33. Javaheri S. Positive airway pressure treatment of central sleep apnea with emphasis on heart failure, opioids, and complex sleep apnea. Sleep Med Clin 2010; 407-417.

34. Fietze I, Ringel D, Glos M, et al. Occurrence and predisposition for complex apneas during CPAP titration in OSA patients. Eur Respir J 2008; 32: Suppl. $52,118 \mathrm{~s}$.

35. Bitter T, Westerheide N, Faber L, et al. Adaptive servoventilation in diastolic heart failure and Cheyne-Stokes respiration. Eur Respir J 2010; 36: 385-392.

36. Kuzniar T, Morgenthaler T. Treatment of complex sleep apnea syndrome. Curr Treatm Options Neurol 2008; 10: 336-341.

37. Glidewell RN, Orr WC, Imes N. Acetazolamide as an adjunct to CPAP treatment: a case of complex sleep apnea in a patient on long-acting opioid therapy. J Clin Sleep Med 2009; 5: 63-64.

38. Kuzniar T, Pusalavidyasagar S, Gay P, et al. Natural course of complex sleep apnea - a retrospective study. Sleep Breath 2008; 12: 135-139.

39. Morgenthaler TI, Gay PC, Gordon N, et al. Adaptive servo-ventilation versus non-invasive positive pressure ventilation for central, mixed, and complex sleep apnea syndromes. Sleep 2007; 30: 468-475.

40. Brown SE, Mosko SS, Davis JA, et al. A retrospective case series of adaptive servoventilation for complex sleep apnea. J Clin Sleep Med 2011; 7: 187-195.

41. Allam JS, Olson EJ, Gay PC, et al. Efficacy of adaptive servoventilation in treatment of complex and central sleep apnea syndromes. Chest 2007; 132: 1839-1846.

42. Javaheri S, Goeeting MG, Khayat R, et al. The performance of two automatic servo-ventilation devices in the treatment of central apnea. Sleep 2011; 34: 1693-1698. 
43. Teschler H, Dohring J, Wang YM, et al. Adaptive pressure support servo-ventilation: a novel treatment for Cheyne-Stokes respiration in heart failure. Am J Respir Crit Care Med 2001; 164: 614-619.

44. Becker M, Tröster N, Scheidl S, et al. Adaptive servoventilation for complex sleep apnea in patients with different underlying diseases. Eur Respir J 2008; 32: Suppl. 52, $743 \mathrm{~s}$

45. Randerath WJ, Galetke W, Stieglitz S, et al. Adaptive servo-ventilation in patients with coexisting obstructive sleep apnoea/hypopnoea and Cheyne-Stokes respiration. Sleep Med 2008; 9: 823-830.

46. Thomas RJ, Daly RW, Weiss JW. Low-concentration carbon dioxide is an effective adjunct to positive airway pressure in the treatment of refractory mixed central and obstructive sleep-disordered breathing. Sleep 2005; 28: 69-77.

47. Dempsey J, skatrud J, Smith C. Powerful stabilizing effects of $\mathrm{CO}_{2}$ during CPAP treatment. Sleep 2005; 28: 12-13.

48. Badr MS, Skatrud JB, Dempsey JA. Effect of chemoreceptor stimulation and inhibition on total pulmonary resistance in humans during NREM sleep. J Appl Physiol 1994; 76: 1682-1692.

49. Gilmartin G, McGeehan B, Bigneault K, et al. Treatment of positive airway pressure treatment associated respiratory instability with enhanced expiratory rebreathing space. J Clin Sleep Med 2010; 6: $529-538$
50. Rapoport D. Stabilizing ventilation in OSAHS with CPAP Emergent periodic breathing through the use of dead space. J Clin Sleep Med 2010; 6 : 539-540.

51. Thomas RJ. Effect of added dead space to positive airway pressure for treatment of complex sleepdisordered breathing. Sleep Med 2005; 6: 177-178

52. Kuzniar TJ, Kovacevic-Ristanovic R, et al. Complex sleep apnea unmasked by the use of a manidbular advancement device. Sleep Breath 2011; 15: 249-252.

53. Guilleminault C, Cummiskey J. Progressive improvement of apnea index and ventilatory response to $\mathrm{CO}_{2}$ after tracheostomy in obstructive sleep apnea syndrome. Am Rev Respir Dis 1982; 126: 14-20.

54. Corcoran S, Mysliwiec V, Niven AS, et al. Development of central sleep apnea after maxillofacial surgery for obstructive sleep apnea. J Clin Sleep Med 2009; 5: 151-153.

55. Goldstein C, Kuzniar TJ. The emergence of central sleep apnea after surgical relief of nasal obstruction in obstructive sleep apnea. J Clin Sleep Med 2012; 8: 321-322.

56. Guilleminault C, Simmons B, Motta J, et al. Obstructive sleep apnea syndrome and tracheostomy. Arch Intern Med 1981; 141: 985-988.

57. Coccagna G, Mantovani M, Brignani F, et al. Tracheostomy in hypersomnia with periodic breathing. Bull Physiopathol Respir (Nancy) 1972; 8: 1217-1227. 